\title{
0 cenário do mercado de trabalho para idosos e a violência sofrida
}

\author{
The labor market scenario for older people and the violence they suffer
}

\section{Resumo}

Objetivo: analisar sistematicamente as publicações referentes ao cenário do mercado de trabalho para idosos e às situações de violência enfrentadas pelos idosos ativos. Método: foi realizada uma revisão integrativa da literatura nas bases de dados Web of Science, Scielo (Scientific Electronic Library Online) PubMed e Science Direct. Resultados: foram encontrados 19 trabalhos, 89,4\% dos casos de origem internacional, sendo 52,6\% com abordagem quantitativa; $31,5 \%$, qualitativa; e, 15,7\%, mista. As áreas do conhecimento que concentraram a maioria dos estudos foram saúde, psicologia e gerontologia com $15,7 \%$, sendo a maior parte dos estudos publicados nos anos de 2019 , com 31,5\% e $26,3 \%$ de recorrências respectivamente. Conclusão: os estudos evidenciaram que os idosos encontram dificuldades de se manterem no mercado de trabalho pelas inadequações do local de trabalho e pela condição de saúde do idoso. Por outro lado, existem iniciativas governamentais que visam melhorar as condições de trabalho dos longevos, sendo a presença do idoso benéfica para sua saúde e também para o mercado. Em relação à violência sofrida no trabalho, os estudos são escassos, e os existentes destacam as dificuldades de entrada e permanência dos longevos no mercado em detrimento do ageismo e dos estereótipos ligados ao envelhecimento. Finalmente, com o envelhecimento da população considera-se importante pensar políticas públicas que proporcionem ao idoso condições adequadas de se manter ativo com a proteção de sua saúde e qualidade de vida.

\section{Abstract}

Objective: to systematically analyze publications referring to the labor market for older people and the violence situations faced by active older people. Method: an integrative literature review in the databases Web of Science, Scielo (Scientific Electronic Library Online) PubMed and Science Direct. Results: 19 papers were found, $89.4 \%$ of the cases of international origin,

\section{Palavras-chave:}

Envelhecimento. Trabalho. Dinâmica Populacional. Maus-Tratos ao Idoso.

Keywords: Aging. Work. Population Dynamics. Elder Abuse.

\footnotetext{
Universidade Federal de Viçosa, Departamento de Economia Doméstica, Programa de Pós-Graduação em Economia Doméstica. Viçosa, MG, Brasil.

Os autores declaram não haver conflito na concepção deste trabalho.

Financiamento: Coordenação de Aperfeiçoamento de Pessoal de Nível Superior (CAPES). N No do processo: 88882.436947/2019-01 Bolsa de: Mestrado 
$52.6 \%$ with a quantitative approach; $31.5 \%$ with a qualitative approach; and $15.7 \%$ with both. The areas of knowledge comprising most of the studies were health, psychology, and gerontology with $15.7 \%$ of them, and most studies were published in 2019, with $31.5 \%$ and $26.3 \%$ of recurrences, respectively. Conclusion: the studies showed that older people have difficulties to remain in the labor market due to the inadequacies of the workplace and their health condition. On the other hand, there are government initiatives aimed at improving the working conditions for older people, and their presence is beneficial both for their health and for the market. Regarding violence at work, studies are scarce, and the existing ones emphasize the difficulties of older people to enter and remain in the market to the detriment of ageism and stereotypes related to aging. Finally, as the population is aging, it is important to think about public policies to provide older people with adequate conditions to remain active and protect their health and quality of life.

\section{INTRODUÇ̃̃O}

O aumento do número de pessoas idosas em todo o mundo reflete em demandas de cunho político, social e econômico e causa reflexos no mercado de trabalho, pois aumenta a presença de idosos na População Economicamente Ativa (PEA) ${ }^{1}$. Nesse contexto, surge a necessidade de mudanças estruturais nas organizações, como, por exemplo, o oferecimento de jornadas de trabalho flexíveis, condições ergonômicas adequadas, gestão de grupos intergeracionais, e, a criação de estratégias para lidar com o ageismo nas empresas ${ }^{2,3}$.

Como o envelhecimento populacional ocorre de forma distinta entre países desenvolvidos e em desenvolvimento ${ }^{4}$, alguns estão mais preparados do que outros para absorver seus trabalhadores idosos. Segundo Cepellos Filho ${ }^{5}$, o mercado de trabalho brasileiro não tem se preparado para abarcar a população de idosos na PEA. Em contrapartida, países desenvolvidos, como a Dinamarca e o Canadá, possuem maiores iniciativas para conter essa parcela populacional ativa, tal como o aumento da idade para aposentadoria e políticas públicas de incentivo à permanência da pessoa idosa no trabalho ${ }^{6}$.

Além disso, o aumento do número de pessoas idosas em relação à população total contribuiu para a elevação do número de casos de violência contra idosos. Segundo Morilla e Manso ${ }^{7}$, a violência contra a pessoa idosa tem se tornado uma tendência mundial, sendo uma realidade preocupante à medida que atinge uma em cada seis indivíduos de 60 anos ou mais ${ }^{8}$.

A violência que atinge os longevos ocorre no ambiente doméstico, na rua e também em instituições, como o mercado de trabalho ${ }^{9}$, podendo causar transtornos físicos, emocionais, psicológicos e provocar efeitos negativos no bem-estar e na qualidade de vida ${ }^{10}$. Segundo Bialowolska et al. ${ }^{11}$ a violência no mercado de trabalho com idosos tem crescido cada vez mais devido às políticas de gestão que visam ao lucro e a redução de custos, deixando questões como as relações entre gerações, que são propulsoras de violência, em segundo plano.

Nesse sentido, levando em consideração a presença cada vez maior de idosos no mercado de trabalho, bem como a crescente onda de violência cometida contra essa parcela da população é que se justifica a necessidade de conhecer o cenário do mercado de trabalho para trabalhadores idosos e as situações de violência por eles enfrentada nesse ambiente.

Dessa forma, este estudo objetivou analisar as publicações referentes ao cenário do mercado de trabalho para idosos e às situações de violência vivenciadas pelos idosos ativos.

\section{MÉTODO}

O presente estudo é uma revisão sistemática de literatura do tipo integrativa. Segundo Botelho et al. ${ }^{12}$ a revisão integrativa possibilita a sistematização do conhecimento científico de um determinado tema, viabiliza a construção do estado da arte sobre o mesmo, além de apontar lacunas da literatura que podem se tornar temas de estudos futuros. Nesse caso, as temáticas de interesse são as violências a que estão sujeitos os idosos economicamente ativos; e, o cenário do mercado de trabalho para trabalhadores idosos. 
A revisão integrativa seguiu as seguintes etapas: escolha da temática de interesse; estabelecimento dos critérios de inclusão e exclusão dos estudos; definição das informações a serem extraídas dos estudos selecionados; avaliação dos estudos que se encaixaram nos critérios de inclusão e exclusão; e, análise, interpretação e apresentação da síntese dos resultados ${ }^{13}$.

Os descritores utilizados para pesquisa foram "Demographic Aging" AND "Violence" AND "Labor" OR "Workers age". A busca foi realizada nas bases de dados Web of Science, Scielo, Pubmed, Science Direct, Scopus, PsychInfo, Jstor, Springer, e Nature.

Os critérios de inclusão utilizados para seleção dos artigos foram aqueles que tratavam da temática em questão, abrangendo as diferentes áreas do conhecimento, com texto completo disponível online, sem filtro de idioma e de ano de publicação. Os critérios de exclusão foram artigos de revisão de literatura, de acesso incompleto e artigos duplicados. As informações extraídas dos textos foram: autores dos artigos, nomes dos periódicos, tipos de estudo, base utilizada para pesquisa, área do conhecimento, país de publicação, ano de publicação, e, os aspectos relacionados ao panorama do mercado de trabalho e às situações de violência vivenciadas pelos longevos ativos. $\mathrm{E}$ por fim, os dados foram organizados por meio de tabelas.

A consulta à literatura foi realizada de agosto de 2020 a janeiro de 2021, mediante o acesso às plataformas PubMed, Science Direct, Web of Science e Scielo, sendo as duas últimas, por meio do login institucional da Universidade Federal de Viçosa no Portal de Periódicos da Capes (Coordenação de Aperfeiçoamento de Pessoal de Nível Superior), utilizando o acesso CAFE (Comunidade Acadêmica Federada), o que permite acesso a artigos de forma completa. Para as demais bases que não possuíam acesso aberto, a busca pelos artigos teve que ser realizada na internet a fim de evitar a perda de conteúdo. Assim sendo, embora tenha se buscado identificar o maior número possível de estudos relacionados à questão proposta, alguns artigos podem ter sido excluídos não intencionalmente, sendo o principal viés deste estudo, o viés de seleção.

\section{RESULTADOS}

Ao realizar a busca nas bases de dados supracitadas, verificou-se que, na Scopus, PsychInfo, Jstor, Springer, e Nature não obteve-se nenhum resultado. E nas demais bases, ao iniciar a busca dos artigos pelo uso dos descritores previamente citados, foram encontrados 780 estudos, dos quais somente 355 deles possuíam acesso aberto. Após selecionar os artigos de acesso livre foi realizada a leitura dos resumos para verificar quais realmente se enquadravam na temática de interesse, sendo descartados 332 estudos que não se adequavam, além de três estudos de revisão de literatura e um artigo duplicado. Finalmente, a amostra foi composta por 19 artigos da literatura nacional e também internacional. Verificou-se, portanto, que a literatura sobre o idoso inserido no mercado de trabalho e as situações de violência a que estão sujeitos é escassa (Quadro 1).

A maioria dos estudos da amostra, 19, foi publicada em periódicos internacionais e, em se tratando das perspectivas dos trabalhos, a maior parte apresentou abordagem quantitativa nove; seis qualitativa; e, três mista.

Em relação à área de conhecimento, as pesquisas que retratam a presença de idosos no mercado de trabalho e as situações de violência por eles enfrentadas perpassam diversas áreas. As mais recorrentes foram saúde, psicologia e gerontologia que apareceram em três estudos, seguido de economia e engenharia, que recorreram em dois estudos. As demais, trabalho social, sociologia, gestão, estudos ambientais, relações industriais e do trabalho e ergonomia estavam presentes em um trabalho cada.

A maior parte das publicações compreenderam os anos de 2019 com seis recorrências e 2020, com cinco, seguido dos anos de 2015, 2016 e 2018 todos com 2 artigos cada, e em 2001 e 1997 obteve-se 1 estudo cada. Em relação às bases de dados, 12 publicações descenderam da Web of Science, uma do Scielo, cinco do Science Direct e 1 da PubMed (Quadro 2).

Em relação à nacionalidade das pesquisas, os Estados Unidos apresentaram seis estudos. A 
Holanda, Brasil, Noruega, Austrália, Dinamarca e Reino Unido apresentaram dois estudos cada. E
Bélgica, Estocolmo, Polônia e Holanda recorreram em uma pesquisa cada (Quadro 3).

Quadro 1. Percurso metodológico percorrido para busca dos artigos na Web of Science, Scielo, PubMed e Science Direct, Scopus, PsychInfo, Jstor, Springer, e Nature.

\begin{tabular}{|c|c|c|c|c|c|c|}
\hline \multicolumn{6}{|c|}{ Descritores= "Demographic Aging" AND "Violence" AND "Labor" OR "Workers age" } & \multirow[t]{2}{*}{ Total } \\
\hline & $\begin{array}{l}\text { Scopus, PsychInfo, Jstor, } \\
\text { Springer, e Nature }\end{array}$ & PubMed & $\begin{array}{l}\text { Science } \\
\text { Direct }\end{array}$ & $\begin{array}{l}\text { Web of } \\
\text { Science }\end{array}$ & Scielo & \\
\hline & 0 & 242 & 248 & 289 & 1 & 780 \\
\hline Acesso livre & 0 & 201 & 23 & 130 & 1 & 355 \\
\hline Fora do tema & 0 & 198 & 16 & 118 & 0 & 332 \\
\hline Artigos de revisão e duplicados & 0 & 2 & 2 & 0 & 0 & 4 \\
\hline Dentro dos critérios & 0 & 1 & 5 & 12 & 1 & 19 \\
\hline
\end{tabular}

Quadro 2. Perfil dos estudos realizados sobre a presença de idosos no mercado de trabalho e a violência enfrentada pelos longevos ativos nos anos de 1997 a 2021.

\begin{tabular}{|c|c|c|c|c|}
\hline Autores & Periódico & Tipo de estudo & $\begin{array}{l}\text { Área do } \\
\text { conhecimento }\end{array}$ & Ano/Base \\
\hline $\begin{array}{l}\text { Damman, e } \\
\text { Henkens }^{14}\end{array}$ & Journal of Applied Gerontology & Qualitativo & Gerontologia & 2020/Web of Science \\
\hline Burmeister et al. $^{22}$ & Journal of Applied Psychology & Quantitativo & Psicologia & 2020/ Web of Science \\
\hline $\begin{array}{l}\text { Straussner e } \\
\text { Senreich }^{23}\end{array}$ & $\begin{array}{l}\text { Nature public Health } \\
\text { Emergency Collection }\end{array}$ & Quantitativo & Trabalho Social & 2020/ Web of Science \\
\hline Oddone $^{31}$ & $\begin{array}{l}\text { Contemporânea-revista de } \\
\text { sociologia da UFSCAR }\end{array}$ & Qualitativo & Sociologia & 2019/ Web of Science \\
\hline Coombe et al. ${ }^{16}$ & Workplace health \& safety & Quantitativo & Saúde & 2019/Web of Science \\
\hline Merkus et al. ${ }^{17}$ & $\begin{array}{l}\text { International archives } \\
\text { of occupational and } \\
\text { environmental health }\end{array}$ & Quantitativo & Saúde & 2019/Web of Science \\
\hline Peters et al. ${ }^{25}$ & Frontiers in psychology & Quantitativo & Psicologia & 2019/ Web of Science \\
\hline Amorim et al. ${ }^{27}$ & Rege-revista de gestão & Qualiquantitativo & Gestão & 2019/Web of Science \\
\hline Sundstrup et al. ${ }^{30}$ & $\begin{array}{l}\text { Occupational and } \\
\text { environmental medicine }\end{array}$ & Quantitativo & Saúde & 2018/Web of Science \\
\hline Talbot et al. ${ }^{32}$ & $\begin{array}{l}\text { International journal of } \\
\text { sustainable transportation }\end{array}$ & Qualiquantitativo & Estudos ambientais & 2016/Web of Science \\
\hline Findsen ${ }^{26}$ & Educational gerontology & Qualitativo & Gerontologia & 2015/Web of Science \\
\hline $\begin{array}{l}\text { Jonsson; } \\
\text { Kielhofner; Borell }{ }^{28}\end{array}$ & $\begin{array}{l}\text { American journal of } \\
\text { occupational therapy }\end{array}$ & Qualitativo & Psicologia & 1997/Web of Science \\
\hline Schreurs et al. ${ }^{29}$ & $\begin{array}{l}\text { SA Journal of Industrial } \\
\text { Psychology }\end{array}$ & Quantitativo & $\begin{array}{l}\text { Relações industriais } \\
\text { e trabalho }\end{array}$ & 2001/Scielo \\
\hline Kerr et al. ${ }^{18}$ & Plos One & Quantitativo & Gerontologia & 2016/Pubmed \\
\hline Bartkowiak et al. $^{24}$ & Journal of Cleaner Production & Qualitativo & Economia & 2020/Science Direct \\
\hline Vigtel $^{21}$ & Labour Economics & Quantitativo & Economia & 2018/Science Direct \\
\hline Dimovski et al. ${ }^{19}$ & Procedia Manufacturing & Quantitativo & Engenharia & 2019/Science Direct \\
\hline Sundstrup et al. ${ }^{20}$ & Safety and Health at Work & Qualiquantitativo & Engenharia & 2020/Science Direct \\
\hline Case et al. ${ }^{15}$ & Procedia Manufacturing & Qualitativo & Ergonomia & 2015/Science Direct \\
\hline
\end{tabular}

Fonte: As autoras, 2021. 
Quadro 3. Países e Principais resultados que subsidiam a resposta dos objetivos, dos estudos realizados sobre a presença de idosos no mercado de trabalho e a violência enfrentada pelos longevos ativos nos anos de 1997 a 2021.

\begin{tabular}{|c|c|c|}
\hline Autores & País & Principais resultados que subsidiam a resposta dos objetivos \\
\hline $\begin{array}{l}\text { Damman e } \\
\text { Henkens }^{14}\end{array}$ & Holanda & $\begin{array}{l}\text { A flexibilização do horário de trabalho é uma forma de manter os idosos } \\
\text { ativos por maior tempo. Porém, ela é diferente entre homens e mulheres. }\end{array}$ \\
\hline Burmeister et al. ${ }^{22}$ & $\begin{array}{l}\text { Estados } \\
\text { Unidos }\end{array}$ & $\begin{array}{l}\text { A presença de funcionários de diferentes faixas-etárias no trabalho } \\
\text { proporciona troca de conhecimento, satisfação e motivação dos trabalhadores. }\end{array}$ \\
\hline $\begin{array}{l}\text { Straussner e } \\
\text { Senreich }^{23}\end{array}$ & $\begin{array}{l}\text { Estados } \\
\text { Unidos }\end{array}$ & $\begin{array}{l}\text { O trabalho se apresenta como benéfico para os trabalhadores idosos, pois } \\
\text { proporciona bem-estar e satisfação. }\end{array}$ \\
\hline Oddone $e^{31}$ & Brasil & $\begin{array}{l}\text { O ageismo é um dos fatores que definem a saída antecipada do idoso do } \\
\text { mercado de trabalho, bem como a saúde econômica das empresas. }\end{array}$ \\
\hline Coombe et al. ${ }^{16}$ & $\begin{array}{l}\text { Estados } \\
\text { Unidos }\end{array}$ & $\begin{array}{l}\text { A saúde do sono dos trabalhadores é um preditor de bom desempenho no } \\
\text { trabalho. }\end{array}$ \\
\hline Merkus et al. ${ }^{17}$ & Noruega & $\begin{array}{l}\text { A condição de saúde do trabalhador idoso pode ser preservada a depender do } \\
\text { esforço exigido pelo trabalho durante toda a vida. }\end{array}$ \\
\hline Peters, et al. ${ }^{25}$ & Austrália & $\begin{array}{l}\text { A estereotipagem negativa do envelhecer no mercado de trabalho } \\
\text { compromete a permanência e/ou retorno do idoso pro trabalho. Dessa forma } \\
\text { julga-se importante que o departamento de recursos humanos se concentre } \\
\text { em questões relativas aos estereótipos do envelhecimento. }\end{array}$ \\
\hline Amorim et al. ${ }^{27}$ & Brasil & $\begin{array}{l}\text { A violência contra idosos no mercado de trabalho pode ser observada na ausência } \\
\text { de práticas gerenciais de contratação e retenção de trabalhadores idosos. }\end{array}$ \\
\hline Sundstrup et al..$^{30}$ & Dinamarca & $\begin{array}{l}\text { A capacidade cognitiva do indivíduo não é um fator que influencia sobre sua } \\
\text { saída do mercado de trabalho. }\end{array}$ \\
\hline Talbot, et al. ${ }^{32}$ & Reino Unido & $\begin{array}{l}\text { Estratégias para diminuir os problemas provenientes do transporte para o } \\
\text { trabalho são importantes para diminuir a evasão de trabalhadores. }\end{array}$ \\
\hline Findsen $^{26}$ & $\begin{array}{l}\text { Estados } \\
\text { Unidos }\end{array}$ & $\begin{array}{l}\text { Estereótipos ligados ao envelhecimento, principalmente relacionado à } \\
\text { capacidade de aprendizagem tem prejudicado a permanência dos idosos no } \\
\text { mercado. Dessa forma iniciativas de aprendizagem entre gerações exerce } \\
\text { papel importante nas práticas gerenciais de idade. }\end{array}$ \\
\hline $\begin{array}{l}\text { Jonsson; Kielhofner; } \\
\text { Borell }^{28}\end{array}$ & Estocolmo & $\begin{array}{l}\text { Além de fatores estruturais e ambientais, a condição de saúde da pessoa idosa } \\
\text { influência sobre a decisão aposentadoria precoce. }\end{array}$ \\
\hline Schreurs et al. ${ }^{29}$ & Bélgica & $\begin{array}{l}\text { Para manter o idoso no mercado de trabalho por mais tempo é necessário que } \\
\text { o trabalho proporcione condições que o mantenha motivado. }\end{array}$ \\
\hline Kerr et al. ${ }^{18}$ & $\begin{array}{l}\text { Estados } \\
\text { Unidos }\end{array}$ & $\begin{array}{l}\text { Diminuir o tempo de ficar sentado durante o trabalho é uma forma de reduzir } \\
\text { a aposentadoria por problemas de saúde. Tendo em vista que diminuindo o } \\
\text { tempo sentado contribui para redução do sedentarismo. }\end{array}$ \\
\hline Bartkowiak et al. ${ }^{24}$ & Polônia & $\begin{array}{l}\text { Os empresários têm mantido seus trabalhadores idosos em sua equipe, } \\
\text { pois reconhecem que eles possuem capital humano e social de fundamental } \\
\text { importância para o mercado. }\end{array}$ \\
\hline Vigtel $^{21}$ & Noruega & $\begin{array}{l}\text { A redução da idade mínima de aposentadoria apresenta efeitos positivos sobre } \\
\text { a contratação de trabalhadores idosos. }\end{array}$ \\
\hline Dimovski et al. ${ }^{19}$ & $\begin{array}{l}\text { Estados } \\
\text { Unidos }\end{array}$ & $\begin{array}{l}\text { A partir da redução da idade mínima de aposentadoria os idosos têm } \\
\text { permanecido por mais tempo no trabalho. }\end{array}$ \\
\hline Sundstrup et al..$^{20}$ & Dinamarca & $\begin{array}{l}\text { A saída precoce de idosos do mercado de trabalho está relacionada a fatores } \\
\text { ambientais, como trabalho duro e exposição a ruídos e poeira. }\end{array}$ \\
\hline Case et al. ${ }^{15}$ & Reino Unido & $\begin{array}{l}\text { A partir da modelagem digital humana é possível verificar se o ambiente de } \\
\text { trabalho é prejudicial à saúde do idoso. E dessa forma pode-se evitar a saída } \\
\text { precoce dele do trabalho por motivos de saúde. }\end{array}$ \\
\hline
\end{tabular}

Fonte: as autoras, 2021. 


\section{DISCUSSÃO}

A partir dos estudos encontrados, verificaram-se as abordagens dadas às pesquisas sobre a temática de interesse. Foram detectados estudos que tratavam de aspectos que influenciam sobre a permanência da pessoa idosa no trabalho, benefícios do trabalho para o trabalhador e para o mercado de trabalho, violências enfrentadas pelo idoso no mercado de trabalho, e, fatores que influenciam na saída precoce dos idosos do mercado do trabalho.

Em relação aos aspectos que influenciam sobre a permanência da pessoa idosa no trabalho em diferentes países, foram identificados sete estudos (Damman e Henkens ${ }^{14}$, Case et al..$^{15}$, Coombe et al. ${ }^{16}$, Merkus et al..$^{17}$, Kerr et al. ${ }^{18}$, Dimovski et al. ${ }^{19}$, Sundstrup et al. ${ }^{20}$, Vigtel $\left.^{21}\right)$. Damman e Henkens ${ }^{14}$ em um estudo realizado na Holanda, ressaltaram que a permanência da pessoa idosa no trabalho está associada às condições de trabalho. Nesse sentido, a fim de oferecer melhores condições e prevenir a saída dos idosos, o governo holandês criou a política de flexibilização do trabalho, oferecendo ao idoso a oportunidade de trabalhar com horários flexíveis ou em sua própria residência.

As condições de trabalho como forma de manutenção do idoso no trabalho também foram mencionadas em estudo realizado no Reino Unido. Este por sua vez assinalou a modelagem digital humana como forma de melhorar as circunstâncias de trabalho dos idosos ${ }^{15}$.

Outro aspecto também mencionado para manutenção do idoso no trabalho é seu estado de saúde. De acordo com Coombe et al. ${ }^{16}$ a condição de saúde, principalmente em relação à saúde do sono do longevo, está atrelada ao seu desempenho e permanência no trabalho. Dessa forma, manter um bom nível de sono dos trabalhadores é uma forma de mantê-los saudáveis e por maior tempo no mercado de trabalho ${ }^{16}$.

A situação de saúde da pessoa idosa também foi mencionada em um trabalho Norueguês realizado por Merkus et al. ${ }^{17}$ que demonstraram que manter a capacidade física do trabalhador durante toda a vida é a chave para a manutenção de uma boa saúde e atividade até o momento da aposentadoria.
Sendo assim, para prolongamento da vida laborativa do idoso é necessário que sejam feitas adaptações em seu trabalho, exigindo menor esforço físico e, consequentemente, provocando menor desgaste em sua saúde ${ }^{17}$.

A saúde mais uma vez foi mencionada como fator importante para manutenção do idoso no trabalho em pesquisa americana realizada por Kerr et al. ${ }^{18}$, na qual demonstrou que o sedentarismo de idosos é um dos maiores causadores de doenças. Sendo assim, buscou-se diminuí-lo por meio da redução de horas diárias sentadas e aumento de transições, como ficar em pé/sentado, a fim de melhorar sua qualidade de vida e consequentemente, prolongar sua vida ativa.

Também preocupado com a vida ativa dos idosos trabalhadores, diante de sua maior presença no mercado e pela escassez de mão de obra, Dimovski ${ }^{19}$ estimou a idade produtiva limítrofe da pessoa idosa, visando o retorno produtivo do idoso para com o trabalho.

Ainda referente à saúde, mas dessa vez, da capacidade cognitiva do idoso, Sundstrup et al. ${ }^{20}$ realizaram um estudo a fim de estimar a relação entre a capacidade cognitiva individual com saídas temporárias ou permanente do mercado. Verificou-se que a capacidade cognitiva nesse caso não interferiu no afastamento do trabalho.

Finalmente, um artigo Norueguês verificou que outro fator que influencia sobre a permanência e contratação de pessoas idosas é a gestão governamental do país, que por meio da legislação vigente pode incentivar as empresas a contratar e manter trabalhadores idosos em suas empresas. A exemplo, a Noruega, ao optar pela flexibilização da idade mínima de aposentadoria, incentivou a maior contratação de idosos no mercado de trabalho ${ }^{21}$.

Outra abordagem encontrada nos estudos trata sobre os benefícios do trabalho para o mercado e para o idoso trabalhador. Com o envelhecimento populacional, ocorrem maiores interações sociais entre pessoas de diferentes idades no mercado de trabalho. Estudo americano desenvolvido por Burmeister et al. ${ }^{22}$ verificou que a presença de pessoas idosas na empresa torna-se vantajosa, pois proporciona troca de conhecimento entre as gerações, 
motiva os funcionários e proporciona maior anseio do idoso em permanecer ativo.

Pesquisa americana desenvolvida por Straussner e Senreich ${ }^{23}$, demonstrou que o trabalho é benéfico para a pessoa idosa, pois, apesar de apresentarem complicações de saúde, que neste caso são provenientes do processo natural do envelhecimento, os idosos se expressam mais satisfeitos com sua profissão, com sua vida e apresentam percepção positiva em relação ao ambiente de trabalho.

A presença de idosos no trabalho ainda se apresenta como benéfica para o mercado, pois segundo Bartkowiak et al. ${ }^{24}$, os gestores de empresas reconhecem que esses trabalhadores podem oferecer para a empresa um bom espírito colaborativo, competências, capital intelectual e social, características que não são encontradas na maioria dos jovens trabalhadores.

Outra categoria encontrada descreve as situações de violência enfrentadas pelos trabalhadores idosos no mercado de trabalho. A violência com o idoso no mercado de trabalho se apresenta por meio do despreparo de empresas para lidar com o novo perfil de trabalhador. Um estudo holandês assinalou que, apesar da conformação etária estar se transformando, tendo cada vez mais idosos em relação a jovens no mercado, a gestão de recursos humanos de empresas não está preparada para lidar com as demandas advindas da convivência intergeracional. Além disso, vê-se que as ações dos gestores são inclinadas à estereotipagem negativa baseada na idade, que considera o idoso um ser improdutivo e incapacitado. Essa estereotipagem prejudica os idosos em termos de oportunidades de emprego e também de se manterem no trabalho ${ }^{25}$.

A violência ancorada nos estereótipos do envelhecimento também foi mencionada em estudo americano. De acordo com Findsen ${ }^{26}$, os preconceitos que circundam o envelhecimento, principalmente os relacionados à capacidade de aprendizagem, têm prejudicado a permanência dos idosos no trabalho. Nesse sentido, considera-se importante que as organizações combatam atitudes de discriminação por idade e incentivem as iniciativas de aprendizagem mútua por meio de atividades intergeracionais ${ }^{26}$.
A violência com os idosos trabalhadores pode ser observada também nas práticas gerenciais. De acordo com Amorim et al. ${ }^{27}$, em um estudo brasileiro, identificaram que empresas que fazem parte do Grupo das Melhores Empresas para se Trabalhar não possuem práticas gerenciais de incentivo à contratação e retenção de trabalhadores idosos. Além disso, não se atentam à adaptação do ambiente e da jornada de trabalho e não possuem planos de saúde com atenção especial às necessidades dos idosos. Ademais, observou-se que os profissionais do Gerenciamento de Recursos Humanos não manifestam interesse na temática, o que se configura mais uma vez como uma violência estrutural ${ }^{27}$.

Outra categoria que emergiu dos estudos, se refere aos fatores que influenciam na saída precoce dos idosos do mercado de trabalho. Jonsson et al. ${ }^{28}$, em estudo realizado em Estocolmo com idosos trabalhadores, revelou que são muitos os fatores internos e ambientais que influenciam sobre a decisão de aposentadoria precoce da pessoa idosa, mas que geralmente os mais influentes são diminuição de energia e doenças crônicas.

Investigação realizada na Bélgica, que examinou os elementos envolvidos nas intenções precoces de aposentadoria, verificou que um dos principais responsáveis pelo anseio de aposentar é o prazer em trabalhar. Neste sentido, verifica-se que manter um funcionário idoso motivado é tão importante quanto mantê-lo com saúde para que ele continue trabalhando ${ }^{29}$.

Outro fator apontado por um estudo dinamarquês como preditor da saída antecipada do idoso do mercado de trabalho é a circunstância do ambiente de trabalho. Segundo Sundstrup et al. ${ }^{30}$, a saída adiantada do mercado de trabalho por trabalhadores idosos está relacionada a fatores ambientais, como as exigências do trabalho físico, levantamento de peso, a exposição a ruídos, poeira e vibração ${ }^{30}$.

As mudanças advindas com a globalização; também apresentam influência sobre a saída precoce do idoso do mercado. Como por exemplo: as atualizações tecnológicas, empregos temporários e contratos a tempo certo, que não proporcionam condições favoráveis de permanência no trabalho. A continuidade do idoso também é influenciada 
pela conjuntura econômica das empresas e de crises macro sistêmicas que as afetam, deixando, portanto, de ser a idade o único demarcador de interrupção da vida ativa do longevo ${ }^{31}$.

No Reino Unido, um estudo verificou a relação entre as intenções precoces de aposentadoria e o transporte até o local de trabalho. Os elementos que mais intervieram sobre a possibilidade de aposentadoria em relação ao transporte foram os altos custos, o estresse, a condição de saúde, o cansaço e o tempo que passam no transporte. Para fazer com que o transporte não influencie sobre a saída do mercado, as empresas têm aplicado estratégias de carona coletiva, vagas de estacionamento reservadas, ajustes do turno de trabalho e trabalho remoto ${ }^{32}$.

De forma semelhante, Nomiyama et al. ${ }^{33}$ verificaram em sua pesquisa realizada no Japão que a forma com que o idoso idealiza o processo de transição para a aposentadoria varia conforme o significado que o trabalho possui em sua vida. Dessa forma, os idosos que veem o trabalho como fardo terão prazer em se aposentar e poder ocupar seu tempo de outras formas. Por outro lado, os idosos que têm o trabalho como parte de sua identidade terão maiores dificuldades de se aposentar. Sendo assim, o significado do trabalho apresenta-se como fator considerável para a decisão de aposentadoria do idoso.

Por fim, cabe ressaltar como limitação do estudo a obtenção incompleta de pesquisas que dizem respeito ao cenário do mercado de trabalho para idosos, bem como de situações de violências ocorridas, devido a indisponibilidade do texto completo online, o que impediu o uso dos mesmos para compor o trabalho.

\section{CONCLUSÕES}

A maior parte dos estudos referentes à presença de idosos no mercado de trabalho e à violência sofrida por eles têm sido realizados em países desenvolvidos, o que pode ser: o processo de envelhecimento gradual ocorrido nesses países tenha proporcionado uma maior amplitude temporal para se pensar e lidar com as questões advindas da longevidade. Acredita-se também que isso pode estar relacionado ao fato de os países desenvolvidos serem os maiores responsáveis pelas publicações científicas em geral. No Brasil, os estudos indicam que os longevos enfrentam dificuldades para a permanência no mercado de trabalho pelas inadequações desse ambiente pela condição de saúde do trabalhador, embora existam exemplos de iniciativas governamentais em outros países para melhorar suas condições de trabalho, como a política de flexibilização do trabalho e a modelagem digital humana. A presença do idoso no trabalho é benéfica tanto para o mercado quanto para o próprio idoso. Quanto à violência sofrida no mercado de trabalho, os estudos são escassos, e os que existem destacam as dificuldades de entrada e permanência dos longevos no mercado devido ao ageismo e aos estereótipos ligados ao envelhecimento. Diante do exposto, considera-se importante pensar em políticas públicas que proporcionem ao idoso condições adequadas de se manter ativo com a proteção de sua saúde e qualidade de vida.

Editado por: Tamires Carneiro de Oliveira Mendes

\section{REFERÊNCIAS}

1. Souza AC, Melo CVB. O mercado de trabalho brasileiro diante das perspectivas de envelhecimento da população. Brasília, DF: Edições Câmara; 2017. Primeira parte, Brasil 2050 desafios de uma nação que envelhece; p. 19-40.

2. Silva ACC, Helal DH. Compreendendo a aposentadoria: um estudo de caso em uma instituição pública do Estado de Pernambuco. Rege. 2017;24:316-24.
3. Zigerr R, Filippim ES, Beltrame V. Perspectivas de carreira para pessoas idosas nas organizações. Recape. 2017;7(3):64-87.

4. Giacomelli GS, Chiapinoto FV, Marion Filho PJ, Vieira KM. Transição demográfica e gasto público: uma análise comparativa de diferentes contextos. Rev Est Soc. 2016;18(37):1-12.

5. Cepellos V, Pereira Filho JL. Envelhecimento nas empresas. Soc Gestão. 2018;17(2):50-9. 
6. Fasbender U, Deller J. Career Management Over the Life-Span. In: Parry E, McCarthy J. The Palgrave Handbook of Age Diversity and Work. London: Palgrave Macmillan; 2016. p. 767.

7. Morilla JL, Manso MEG. Violência contra pessoa idosa: contribuições para o estudo do tema. Rev Longeviver. 2020;2(6):93-9.

8. Yongjie Y, Christopher RM, Zachary DG, Kathleen HW. Elder abuse prevalence in community settings: a systematic review and meta-analysis. Lancet Glob Health. 2017;5(2):147-56.

9. Santos MAB, Moreira R, Faccio PF, Gomes GC, Silva VL. Fatores associados à violência contra o idoso: uma revisão sistemática da literatura. Ciênc Saúde Colet. 2020;25(6):2153-75.

10. World Health Organization. Elder Abuse: the health sector role in prevention and response. Geneva: WHO; 2016.

11. Bialowolska B, Bialowolska D, McNeely E. The impact of workplace harassment and domestic violence on work outcomes in the developing world. W Devel. 2020;126:1-11.

12. Botelho LLR, Cunha CCA, Macedo M. O método da revisão integrativa nos estudos organizacionais. Gestão Soc. 2011;5(11):121-36.

13. Mendes KDS, Silveira RCCP, Galvão CM. Revisão integrativa: método de pesquisa para a incorporação de evidências na saúde e na enfermagem. Texto Contexto Enferm. 2008;17(4):758-64.

14. Damman M, Henkens K. Gender differences in perceived workplace flexibility among older workers in the Netherlands: a brief report. J Appl Gerontol. 2020;39(8):915-21.

15. Case K, Hussain A, Marshall R, Summerskill S. Digital human modelling and the ageing workforce. Procedia Manuf. 2015;3(1):3694-3701.

16. Coombe AH, Epps F, Lee J, Chen ML, Imes CC, Chasens ER. Sleep and self-rated health in an aging workforce. Workplace Health Saf. 2019;67(6):302-10.

17. Merkus SL, Lunde LK, Koch M, Waersted M, Knardahl S, Veiersted KB. Physical capacity, occupational physical demands, and relative physical strain of older employees in construction and healthcare. Int Arch Occup Environ Health. 2019;92(3):295-307.

18. Kerr J, Takemoto M, Bolling K, Atkin A, Carlson J, Rosenberg D, et al. Two-arm randomized pilot intervention trial to decrease sitting time and increase sit-to-stand transitions in working and non-working older adults. Plos ONE. 2016;11(1):1-12
19. Dimovski V, Grah B, Colnar S, Bogotaj D. Age management of industrial workers based on multiple decrease modeling. Procedia Manuf. 2019;39(1):1455-63.

20. Sundstrup E, Hansen A, Mortensen EL, Poulsen OM, Claussen T, Rugulies R, et al. Cognitive ability in midlife and labor market participation among older workers: prospective cohort study with register follow-up. Saf Health Work. 2020;11(3):291-300.

21. Vigtel TC. The retirement age and the hiring of senior workers. Labour Econ. 2018;51(1):247-70.

22. Burmeister A, Wang M, Hirschi A. Understanding the motivational benefits of knowledge transfer for older and younger workers in age-diverse coworker dyads: an actor-partner interdependence model. Appl Psychol. 2020;105(7):748-59.

23. Straussner SLA, Senreich E. Productive aging in the social work profession: a comparison of licensed workers 60 years and older with their younger counterparts. Clin Soc Work J. 2020;48(2):196-210.

24. Bartkowiak G, Krugiełka A, Kostrzewa-Demczuk P, DachowskI R, Galek K. Attitudes of polish entrepreneurs towards $65+$ knowledge workers in the context of their pro-social attitude and organizational citizenship behavior.sustain. 2020;12(13):1-24 .

25. Peters P, Van der heijden BIJM, Spurk D, de vos A, Klaassen R. Please don't look at me that way. An empirical study into the effects o age-based (meta-) stereotyping on employability enhancement among older supermarket workers. Front Psychol. 2019;10(249):1-14.

26. Findsen B. Older workers' learning within organizations: issues and challenges. Educ Gerontol. 2015;41(8):582-9.

27. Amorim W, Fischer A, Fevorini FB. Workers age 50 and over in the Brazilian labor market: is there ageism? Rege. 2019;26(2):161-79.

28. Jonsson H, Kielhofner G, Borell L. Anticipating retirement: the formation of narratives about an occupational transition. Am J Occup Ther. 1997;51(1):49-56

29. Schreurs B, Cuyper ND, Emmerik IJH, Notelaers G, Witte HD. Job demands and resources and their associations with early retirement intentions through recovery need and work enjoyment. SA J Industr Psychol. 2001;37(2):63-73.

30. Sundstrup E, Hansen AM, Mortensen EL, Poulsen OM, Clausen T, Rugulies R, et al. Retrospectively assessed physical work environment during working life and risk of sickness absence and labour market exit among older workers. Occup Environ Med. 2018;75(2):114-23. 
31. Oddone MJ. Employment, the (de) chronologization of life cycle, and the career path of older workers. Contemporanea. 2019;9(3):803-22.

32. Talbot R, Rackliff L, Nicolle C, Maguire M, Mallaband R. Journey to work: Exploring difficulties, solutions, and the impact of aging. Int J Sustain Transp. 2016;10(6):541-51.
33. Nomiyama T, Omae K, Tanaka S, Miyauchi H, Koizumi A, Tsukada M, et al. A cross-sectional observation of the effects of hydrazine hydrate on workers' health. J Occup Health Psychol. 1998;40(3):177-85. 